\title{
Chemical Composition and Pharmacological Potential of Rudbeckia hirta L. Review
}

\section{Raman Lukashou* and Natalia Gurina}

Organization Pharmacy Department, Belarusian State Medical University, Minsk, Republic of Belarus

*Corresponding Author: Raman Lukashou, Organization Pharmacy Department, Belarusian State Medical University, Minsk, Republic of Belarus.

Received: July 25, 2019; Published: September 13, 2019

DOI: 10.31080/ASMS.2019.03.0409

\begin{abstract}
The article presents systematized scientific data on the chemical composition and pharmacological properties of Rudbeckia hirta L. This plant contains various groups of biologically active substances: phenolic compounds (flavonoids, anthocyanins, hydroxycinnamic acids), acetylene derivatives, etc. In the preclinical models, an immunomodulatory, anti-inflammatory and antioxidant effect is established. Among the flavonoids, patulitrin is considered the dominant. The immunomodulatory and antioxidant effect is associated with flavonoids and hydroxycinnamic acids, and the anti-inflammatory effect is associated with sequestepenoids.
\end{abstract}

Keywords: Rudbeckia hirta; Chemical Composition; Pharmacological Properties

\section{Introduction}

Currently, herbal medicines with an immunomodulatory effect are mainly obtained from plants of the genus Echinacea Moench. Long-term use (more than eight weeks) of these dosage forms is not recommended in view of the occurrence of cross-resistance between various forms obtained on the basis of Echinacea and depletion of the cellular level of immunity.

A promising plant with potential immunomodulatory properties is Rudbeckia hirta L. However, its chemical composition and pharmacological properties are poorly understood. The literature provides fragmentary information on the chemical composition and pharmacological properties of Rudbeckia hirta L. Therefore, the purpose of this article is to systematize the scientific data about chemical composition and pharmacological properties of various parts of Rudbeckia hirta L. and its individual chemicals.

\section{Flavonoids}

Petals of Rudbeckia hirta L. contain flavonols patulitrin, quercetagetin and 6,7-dimethoxy-3',4',5-trihydroxyflavon-3-0-gluco- side [1]. The herb Rudbeckia hirta L. contains quercetahetin-7-0galactoside and patuletin-7-0-galactoside [2].

In 1993, a group of authors published a paper [3] containing information that the composition of the aerial part (herb) of Rudbeckia hirta L., in addition to the flavonoids listed above, includes aglycone eupatolitin, flavonol glycosides (quercetin-7-0-glucoside, quercetagytin-7-0-glucoside) and methoxy derivative of quercetin (eupatolin), as well as dimethoxy-3,5,4'-trihydroxyflavone-30 -ramnoside. The flavonoid composition of flowers and leaves of Rudbeckia hirta L. is identical.

The content of patulitrin in flowers is $1,90 \%$, in leaves $-0,82 \%$ and in stems $-0,12 \%$ [4]. Only patulitrin was found in stem culture. The herb of Rudbeckia hirta L. includes hyperoside and rutin [5].

The flavonoid composition of the petals of Rudbeckia hirta L. is most fully described in [6] and additionally includes: kaempferol and its 3-0- and 7-0-glucosides; 6-methoxykaempferol; 7-methoxykaempferol; 6-hydroxykaempferol; 7,8-dime- 
thoxykaempferol-3-0-glucoside; quercetin and its 3-0-glucoside; 7-methoxyquercetin and quercethagetin-3-0-glucoside. Additionally, eupatolitin-3-0- $\beta$-D-(6"-0-acetyl)-glucopyranoside was found in the petals. The total content of flavonoids in the petals of Rudbeckia hirta L. is $4,72 \%$ [7].

The flowers of Rudbeckia hirta L. contain gossipetin-7-0- $\beta$ glucopyranoside, quercethagetin-7-0- $\beta$-D-glucopyranoside, eupatolin, patulitrin and chrysosplenol D [8].

The figure shows the structural formula of the dominant flavonoid of Rudbeckia hirta L. - patulitrin.

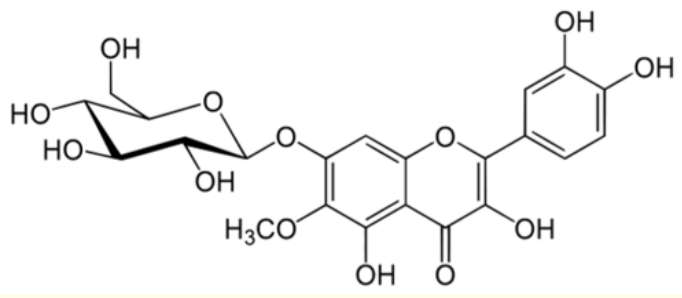

Figure 1: The structural formula of patulitrin [6].

The chemical name of this compound is 3,5,3,4-tetrahydroxy-6methoxyflavone-7-0- $\beta$-D-glucopyranoside. Patulitrin is patuletin 7-0-glucoside, and contains in its molecule a methoxyl group in the 6 th position of the flavan structure.

In the leaves of Rudbeckia hirta L., grown under the conditions of the Central Botanical Garden of the National Academy of Sciences of Belarus, flavonoids accumulate in the amount of about $2,4 \%$ [9]. The content of flavonoids in the leaves of a plant harvested in Slovakia is about $1,2 \mathrm{mg} / \mathrm{kg}$, anthocyanins - about $0,1 \mathrm{mg} / \mathrm{kg}$ [10].

Flavonoids play a significant role in attracting insects for pollination of Rudbeckia hirta L. and are contained in morphological structures that glow when the inflorescence is irradiated with UVlight [11].

\section{Hydroxycinnamic acids}

Rudbeckia hirta L. incorporates hydroxycinnamic acids, the maximum content of which is determined in the inflorescences in the flowering phase and is about 2,3\% (stationary irradiation with white light only) in terms of environmentally friendly biomass of a plant grown under artificial conditions in order to obtain a functional supplement. The content increases with alternating irradiation with white and blue light to almost $3 \%$. In leaves, the content is about $2 \%$, in the stems - about $0,8 \%[12,13]$.

The methanol extract obtained from the frozen leaves of Rudbeckia hirta L. contains various esterification products of quinic acid with caffeic acid [14].

The leaves of Rudbeckia hirta L., harvested in Belarus in different phases of plant development, contain from 1,93 to $4,82 \%$ of the sum of hydroxycinnamic acids and about $1,74 \%$ of the amount of chlorogenic acids [15]. The aerial part of Rudbeckia hirta L. contains caffeic and chlorogenic acids [5]. The flowers of Rudbeckia hirta L. include $\beta$-resorcilic, p-coumaric, caffeic, 5-0-caffeic quinic acids, as well as 5-0-p-cumaroyl quinicacid and its methyl ester [8].

\section{Anthocyanins}

The anthocyanin complex of the callus culture obtained from the flowers of Rudbeckia hirta L. includes cyanidin, its glycosides and ether; delphinidine and its ether; malvidin and petunidine glycoside. The total content of these compounds in tissue culture is $1,6 \%$, the content of cyanidin-3-0-glucoside $-0,4 \%$. In the tubular flowers of an intact plant, only cyanidin-3-0-glucoside is detected in an amount of about $0,3 \%$ in terms of dry raw material [16-18]. The content of anthocyanins in the leaves is about $0,86 \%$, leucoanthocyanidins $-0,46 \%$, catechins $-0,44 \%$ [15].

\section{Acetylene derivatives}

The underground organs of Rudbeckia hirta L. include in their composition three groups of acetylene derivatives: polyacetylenes, tiarubrins and substances containing, in their structure, in addition to the acetylene part, the thiophene cycle (thiophenes). Compounds of all these groups are extracted from plant materials with non-polar solvents [19].

The roots of the plant contain pentaenen; tiarubrin C; 1-tridecene-3,5,7,9,11-pentaen-tiarubrin; 3-(1-propyl)-6-(3,5-hexadien1-inil)-1,2-dithiocyclohexane-3,5-diene and 2-(1-propyl)-6-(3,5hexadiene-1-inil) -thiophene [20,21]. 
The most studied polyacetylene of Rudbeckia hirta L. is pentaenene, for which the laws of biosynthesis, the optimal conditions for the isolation and quantitative determination have been revealed $[22,23]$.

\section{Other groups of biologically active substances}

The fractions obtained by extracting the sum of substances from the inflorescences of Rudbeckia hirta L. with non-polar solvents and their mixtures contain ketones, esters, lactones and fatty acids, as well as minor volatile components [24].

Vegetative and generative organs of Rudbeckia hirta L. grown under artificial conditions include water-soluble sugars, ascorbic acid, and carotenoids [13].

The leaves of Rudbeckia hirta L. contain carotenoids, chlorophylls $a$ and $b$, albumin, globulins, gluteins and prolamins [9].

In callus and suspension cultures of the roots of Rudbeckia hirta L., a representative of the eudesmanolidide group, Pulhelin E, was found, which is absent in the intact plant [25]. The roots of Rudbeckia hirta L., which grow in urbanized areas, accumulate cadmium [26].

Achenes of Rudbeckia hirta L. are rich in fatty acids: palmitic, oleic and linoleic [27]. In transition to flowering, an increase in the concentration of biogenic amines, spermidine and putrescine, is observed in Rudbeckia hirta L. [28].

False-tongued flowers of Rudbeckia hirta L. contain yellow pigments [29], which are used for dyeing fabrics [30].

The roots and grass of Rudbeckia hirta L. contain lectins and alkylamides [5]. The flowers of Rudbeckia hirta L. include the rudbeckolide-sesquiterpene lactone [31].

\section{Pharmacological properties}

The natives of North America used the decoction of the roots of Rudbeckia hirta L. for colds and helminthic invasions; rinses were used topically to treat wounds, ulcerative skin lesions and snake bites; root juice - for the treatment of earache, edema and dropsy [32,33].
In the United States, tincture and essence from the fresh aerial parts of Rudbeckia hirta L. are used in homeopathy for the treatment of colds in children, in urological and gynecological practice, and also in cardiology [34].

Arabinogalactan protein (lectin) from Rudbeckia hirta L. possesses immunomodulatory properties identified in induced with phytohemagglutinin, concanavalin A (T-cell mitogens) and Escherichia coli lipopolysaccharide (B-cell mitogen) leucocyte blast transformation, and the reaction induction Escherichia coli lipopolysaccharide synthesis of immunoglobulin class $M$ using cells of mice. Arabinogalactan-protein from Rudbeckia hirta L. enhances the secretion of granulocyte-macrophage colony-stimulating factor, tumor necrosis factor- $\alpha$, interleukin- 6 and interferon- $\gamma$, and also activates the complement system in the classical and alternative ways. The immunomodulatory effect is dose-dependent [5].

Methanol extraction from flowers and individual components of Rudbeckia hirta L. exhibit immunomodulatory (in phytohemagglutinin-induced leucocyte blast transformation on mouse splenocytes), antioxidant (on the model of Oxygen Radical Absorbance capacity) [8] and anti-inflammatory (inhibit 5-lipoxygenase) properties [31].

Individual components of the methanolic extract: p-coumaric and caffeic acid gossipetin-7-0- $\beta$-glucopyranoside, quercethagetin7-0- $\beta$-D-glucopyranoside, patulitrin, chryzosplenol-D decrease proliferation of lymphocytes stimulated with phytohemagglutinin, from $100 \%$ to $83-92 \%$ (in the negative control, where dexamethasone is added, the decrease occurs to 24\%). Methanol extraction significantly increases it to $130 \%$ (in the positive control, where Echinacea purpurea L. is added, the increase occurs to $142,5 \%$ ). $\beta$-resorcilic acid and eupatolin slightly increase proliferation to $102-105 \%$ [31].

The antioxidant properties of the extracts obtained by extraction with petroleum ether, chloroform, ethyl acetate, n-butanol, ethanol, and individual flavonoids (eupatolitin, eupatolitin-30 - $\beta$-D-glucopyranoside and eupatolitin-3-0- $\beta$-D-(6"-0-acetyl)glucopyranoside) using the $\mathrm{K}_{3}\left[\mathrm{Fe}(\mathrm{CN})_{6}\right]$-method (in comparison with ascorbic acid), 2,2-diphenyl-1-picrylhydrazyl, hydroxyl radicals $(\mathrm{OH} \cdot)$ and superoxide anion (). All extracts exhibit antioxidant 
properties, the ethyl acetate fraction and n-butanol extraction, as well as eupatolitin-3-0-glucoside and eupatolitin-3-0-(6"'-0acetyl)-glucoside, have the greatest antioxidant effect [35].

Chloroform extraction, obtained from callus Rudbeckia hirta L., shows an immunostimulating effect by increasing the production of interferon [36].

There is no cross-immunological stability between the Echinacea purpurea lectin complex and arabinogalactan- Rudbeckia hirta protein [5].

Tiarubrin C, isolated from the roots of Rudbeckia hirta L., exhibits pronounced antibacterial and antifungal properties and can be used as an antiseptic [19].

Extractions from the roots of plants containing tiarubrin $\mathrm{C}$ or pentaenen have insecticidal and anthelmintic properties [37-40].

Polyacetylenes and thiophenes of Rudbeckia hirta L. have a photosensitizing effect $[37,41]$.

\section{Conclusion}

Rudbeckia hirta L. contains in its composition phenolic compounds (flavonoids, hydroxycinnamic acids, anthocyanins), acetylene derivatives and other biologically active substances. Rudbeckia hirta L. has been used in traditional medicine in North America, homeopathy. The plants, its extracts and chemicals isolated from it exhibit an immunomodulatory, anti-inflammatory and antioxidant effect.

The complex of biologically active substances found in the plant and its pharmacological properties provide the basis for further in-depth study of Rudbeckia hirta L. and its application along with Echinacea purpurea L. in medicine.

\section{Bibliography}

1. Thompson W., et al. "Flavonols: pigments responsible for ultraviolet absorption in nectar guide of flower". Science 177.48 (1972): 528-530.

2. Moubasher MH. "Flavonoid investigations of members of Rudbeckia, Echinacea and Dracopis tribe Heliantheae: Asteraceae". Ph. D. thesis (1989).
3. Cisowski W., et al. "Flavonoids from Rudbeckia hirta L. herb". Polish Journal of Chemistry 67 (1993): 829-836.

4. Luczkiewic M., et al. "High-performance liquid chromatographic determination of polymethoxylated flavonols in soilgrowth plants and in vitro cultures Rudbeckia hirta L". Acta Poloniae Pharmaceutica 55.2 (1998): 143-147.

5. Nemetz J. "Untersuchungen zu Inhaltsstoffen von Rudbeckia hirta L. sowie zu deren immunmodulatorischer Wirkung und Bioverfügbarkeit". Dis Doctorgrades der MathematischNaturwissenschaftlichen Fakultät (2004).

6. Schlangen K., et al. "Formation of UV-honey guides in Rudbeckia hirta”. Phytochemistry 70.7 (2009): 889-898.

7. Study on the extraction and antioxydation properties total flavonoids from Rudbeckia hirta petals (2015).

8. Michael BR., et al. "Evidence-based medicinal value of Rudbeckia hirta L. flowers". Natural Product Research 28.12 (2014): 909-913.

9. Kabushava IN. Ecological and biological features of representatives of the genera Echinacea Moench introduced in the conditions of Belarus and Rudbeckia L (2007).

10. Baranyiova I and Brestic M. "Non-destructive analysis of the content of anthocyanins and flavonoids in plants". Proceedings of International PhD Students Conference (2013): 629633.

11. Ferreira MM., et al. "Flavonas e flavonois: novas descobertas sobre sua estrutura quimicae funcao biologica". Revista Agro@mbiente 2.2 (2008): 57-60.

12. Doyko IV., et al. "Dynamics of accumulation of hydroxycinnamic acids in different parts of the plant Echinacea purpurea and hairy ore beak grown in light-culture conditions". Chemistry of Plant Raw Materials 3 (2002): 35-37.

13. Doyko IV. "Effect of spectral irradiation regimes on the biochemical composition of ecologically pure biomass of Echinacea purpurea L. and Rudbeckia hirta L. in light-culture conditions and its use as a functional additive". Databases and their implications in biological Sciences (2003). 
14. Jaiswal R., et al. "Profiling the chlorogenic acids of Rudbeckia hirta, Helianthus tuberosus, Carlina acaulis and Symphyotrichum novae angliae leaves by LC-MSn". Phytochemical Analysis 22 (2011): 432- 441.

15. Hetko NV and Kabushava IN. "Comparative anatomical, physiological and biochemical researches of assimilating organs of Purple Counflower (Echinacea purpurea (L.) Moench)". Sodinikystè ir daržininkystė 19.3 (2000): 362-369.

16. Luczkiewcz M and Cisowski W. "Optimization of the second phase of a two phase growth system for anthocyanin accumulation in callus cultures of Rudbeckia hirta". Plant cell, tissue and organ culture 65.1 (2001): 57-68.

17. Luczkiewcz M and Cisowski W. "Stimulation of anthocyanin production by two-phase growth system in callus of Rudbeckia hirta". Biotechnologia 4 (1999): 199-210.

18. Luczkiewcz M and Cisowski W. "The RP-HPLC Analysis of Anthocyanins". Chromatographia 48.5/6 (1998): 360-364.

19. Guillet G., et al. "Multiple Modes of Insecticidal Action of Three Classes of Polyacetylene Derivatives from Rudbeckia hirta". Phytochemistry 46 (1997): 495-498.

20. Constrabel C., et al. "Dithiacyclohexadienes and thiophenes of Rudbeckia hirta". Phytochemistry 27. 11 (1988): 3533- 3535.

21. Constrabel $\mathrm{C}$ and Towers G. "The complex nature of the mechanism of toxicity of antibiotic dithiacyclohexadiene polyines (thiarubrines) from the astera" Planta Medica 55.1 (1989): 35-37.

22. Almeida-Cortez J., et al. "Effects of nutrient availability on the production of pentaynene, a secondary compound related to defense, in Rudbeckia hirta". Plant Species Biology 18.2/3 (2003): 85-89.

23. Almeida-Cortez J., et al. "Growth and chemical in relation to resource availability: tradeoffs or common responses to environmental stress?". Brazilian Journal of Biology 64 (2004): 187-194.

24. Judkins RN. "Extraction of potential chemical attractants from Rudbeckia hirta inflorescences". Thesis of Master of Sciences (2009).
25. Luczkiewic M., et al. "Production of pulchelin E in hairy roots, callus and suspension cultures of Rudbeckia hirta L". Plant Science 163 (2000): 91-100.

26. Milles LJ and Parker GR. "Effect of soil Cd addition on germination of native plant species". Plant and Soil 54 (1980): 243-247.

27. Cisowski W. et al. "Gas-liquid Chromatographic Analysis of the Fatty Acids Obtained from the Fruit of Some Asteraceae plants". Acta Chromatographica 6 (1996): 215-223.

28. Harkess RL., et al. "Floral morphogenesis in Rudbeckia hirta in relation to polyamine concentration". Physiologia plantarum 86 (1992): 575-582.

29. Stephens SG and Blakeslee AF. "Pigments of yellow-eyed races of the Black-eyed Susan (Rudbeckia hirta)". Proceedings of the National Academy of Sciences 4 (1948): 252-258.

30. Nuffer B. "Black-eyed Susan (Rudbeckia hirta)". 2007.

31. Michael BR., et al. "A new highly oxygenated pseudoguaianolide with 5-LOX inhibitory activity from Rudbeckia hirta L. flowers". Natural Product Research 27.24 (2013): 2281-2285.

32. Church B. "Medicinal plants, trees and shrubs of Appalachia: a field guide" 2006.

33. Moerman DE. "Native American Ethnobotany" 2004.

34. Elston CD. “15 Prairie Flowers of West Michigan” 2000.

35. Study on the extraction and antioxydation properties total flavonoids from Rudbeckia hirta petals (2015).

36. Skwarek T and i dr. "Dzialanie interferogenne hodowli kalusa R. hirta L". XXIII Zjazd Polskiego Towarzystwa Microbiolgicznegj (1996): 53.

37. Guillet G., et al. "Behavioral Adaptations of Two Phytophagous Insects Feeding on Two Species of Phototoxic Asteraceae". Journal of Insect Behavior 8.4 (1995): 533-546.

38. Chitwood DJ. "Phytochemical Based Strategies for Nematode Control". Annual Review of Phytopathology 40 (2002): 221249. 
39. Potter JW and McKeown AW. "Inhibition of Pratylenchus penetrans by intercropping of Rudbeckia hirta and Lycopersicon esculentum in pot cultivation". Phytoprotection 83.2 (2002: 115-120.

40. Sánchez S., et al. "The potential of thriarubrine $\mathrm{C}$ as a nematicidal agent against plant-parasitic nematodes". Journal of Nematology 30 (1998): 192-200.

41. Nelson LS., et al. "Handbook of poisonous and injurious plants" (2007).

Volume 3 Issue 10 October 2019

(C) All rights are reserved by Raman Lukashou and Natalia Gurina. 